\title{
Growth Analysis of Wronskians in terms of Slowly Changing Functions
}

\author{
Sanjib Kumar Datta, ${ }^{1}$ Tanmay Biswas, ${ }^{2}$ and Sultan $\mathrm{Ali}^{3}$ \\ ${ }^{1}$ Department of Mathematics, University of Kalyani, Kalyani, Nadia, West Bengal 741235, India \\ ${ }^{2}$ Rajbari, Rabindrapalli, RN. Tagore Road, Krishnagar, Nadia, West Bengal 741101, India \\ ${ }^{3}$ Department of Mathematics, Kalna College, Kalna, Burdwan, West Bengal 713409, India
}

Correspondence should be addressed to Sanjib Kumar Datta; sanjib_kr_datta@yahoo.co.in

Received 12 August 2012; Accepted 29 September 2012

Academic Editor: Vladislav Kravchenko

Copyright (C) 2013 Sanjib Kumar Datta et al. This is an open access article distributed under the Creative Commons Attribution License, which permits unrestricted use, distribution, and reproduction in any medium, provided the original work is properly cited.

In the paper we establish some new results depending on the comparative growth properties of composite entire or meromorphic functions using generalised $L^{*}$-order and generalised $L^{*}$-type and Wronskians generated by one of the factors.

\section{Introduction, Definitions, and Notations}

We denote by $\mathbb{C}$ the set of all finite complex numbers. Let $f$ be a meromorphic function defined on $\mathbb{C}$. We use the standard notations and definitions in the theory of entire and meromorphic functions which are available in [1] and [2]. In the sequel we use the following notation: $\log ^{[k]} x=$ $\log \left(\log ^{[k-1]} x\right)$ for $k=1,2,3, \ldots$ and $\log ^{[0]} x=x$.

The following definitions are well known.

Definition 1. A meromorphic function $a=a(z)$ is called small with respect to $f$ if $T(r, a)=S(r, f)$.

Definition 2. Let $a_{1}, a_{2}, \ldots a_{k}$ be linearly independent meromorphic functions and small with respect to $f$. We denote by $L(f)=W\left(a_{1}, a_{2}, \ldots, a_{k} ; f\right)$ the Wronskian determinant of $a_{1}, a_{2}, \ldots, a_{k}, f$ that is,

$$
L(f)=\left|\begin{array}{ccccc}
a_{1} & a_{2} & \ldots & a_{k} & f \\
a_{1}^{\prime} & a_{2}^{\prime} & \ldots & a_{k}^{\prime} & f^{\prime} \\
\cdot & \cdot & \cdots & \cdot & \cdot \\
\cdot & \cdot & \cdots & \cdot & \cdot \\
\cdot & \cdot & \cdots & \cdot & \cdot \\
a_{1}^{(k)} & a_{2}^{(k)} & \cdots & a_{k}^{(k)} & f^{(k)}
\end{array}\right| .
$$

Definition 3. If $a \in \mathbb{C} \cup\{\infty\}$, the quantity

$$
\begin{aligned}
\delta(a ; f) & =1-\limsup _{r \rightarrow \infty} \frac{N(r, a ; f)}{T(r, f)} \\
& =\liminf _{r \rightarrow \infty} \frac{m(r, a ; f)}{T(r, f)}
\end{aligned}
$$

is called the Nevanlinna deficiency of the value "a."

From the second fundamental theorem it follows that the set of values of $a \in \mathbb{C} \cup\{\infty\}$, the quaintity for which $\delta(a ; f)>0$ is countable and $\sum_{a \neq \infty} \delta(a ; f)+\delta(\infty ; f) \leq 2(\mathrm{cf}$. [1, page 43]). If, in particular, $\sum_{a \neq \infty} \delta(a ; f)+\delta(\infty ; f)=2$, we say that $f$ has the maximum deficiency sum.

Let $L=L(r)$ be a positive continuous function increasing slowly, that is, $L(a r) \sim L(r)$ as $r \rightarrow \infty$ for every positive constant $a$. Singh and Barker [3] defined it in the following way.

Definition 4 (see [3]). A positive continuous function $L(r)$ is called a slowly changing function if, for $\varepsilon(>0)$,

$$
\frac{1}{k^{\varepsilon}} \leq \frac{L(k r)}{L(r)} \leq k^{\varepsilon}, \quad \text { for } r \geq r(\varepsilon) \text { and }
$$

uniformly for $k(\geq 1)$. 
If further, $L(r)$ is differentiable, the above condition is equivalent to

$$
\lim _{r \rightarrow \infty} \frac{r L^{\prime}(r)}{L(r)}=0 .
$$

Somasundaram and Thamizharasi [4] introduced the notions of $L$-order and $L$-type for entire functions. The more generalised concept for $L$-order and $L$-type for entire and meromorphic functions are $L^{*}$-order and $L^{*}$-type, respectively. In the line of Somasundaram and Thamizharasi [4], for any positive integer $m$ one may define the generalised $L^{*}$-order $\rho_{f}^{[m] L^{*}}$ (generalised $L^{*}$-lower order $\lambda_{f}^{[m] L^{*}}$ ) and generalised $L^{*}$-type $\sigma_{f}^{[m] L^{*}}$ in the following manner.

Definition 5. The generalised $L^{*}$-order $\rho_{f}^{[m] L^{*}}$ and the generalised $L^{*}$-lower order $\lambda_{f}^{[m] L^{*}}$ of an entire function $f$ are defined as

$$
\begin{aligned}
& \rho_{f}^{[m] L^{*}}=\limsup _{r \rightarrow \infty} \frac{\log ^{[m+1]} M(r, f)}{\log \left[r e^{L(r)}\right]}, \\
& \lambda_{f}^{[m] L^{*}}=\liminf _{r \rightarrow \infty} \frac{\log ^{[m+1]} M(r, f)}{\log \left[r e^{L(r)}\right]} .
\end{aligned}
$$

When $f$ is meromorphic, it can be easily verified that

$$
\begin{aligned}
& \rho_{f}^{[m] L^{*}}=\limsup _{r \rightarrow \infty} \frac{\log ^{[m]} T(r, f)}{\log \left[r e^{L(r)}\right]}, \\
& \lambda_{f}^{[m] L^{*}}=\liminf _{r \rightarrow \infty} \frac{\log ^{[m]} T(r, f)}{\log \left[r e^{L(r)}\right]} .
\end{aligned}
$$

Definition 6. The generalised $L^{*}$-type $\sigma_{f}^{[m] L^{*}}$ of an entire function $f$ is defined as follows:

$$
\sigma_{f}^{[m] L^{*}}=\limsup _{r \rightarrow \infty} \frac{\log ^{[m]} M(r, f)}{\left[r e^{L(r)}\right]^{\rho_{f}^{[m] L^{*}}}}, \quad 0<\rho_{f}^{[m] L^{*}}<\infty .
$$

For meromorphic $f$,

$$
\sigma_{f}^{[m] L^{*}}=\limsup _{r \rightarrow \infty} \frac{\log ^{[m-1]} T(r, f)}{\left[r e^{L(r)}\right]^{\rho_{f}^{[m] L^{*}}}}, \quad 0<\rho_{f}^{[m] L^{*}}<\infty .
$$

For $m=1$, we may get the classical cases $\{\mathrm{cf}$. [4] $\}$ of Definitions 5 and 6, respectively.

Lakshminarasimhan [5] introduced the idea of the functions of $L$-bounded index. Later Lahiri and Bhattacharjee [6] worked on the entire functions of $L$-bounded index and of nonuniform $L$-bounded index. Since the natural extension of a derivative is a differential polynomial, in this paper we prove our results for a special type of linear differential polynomials, namely, the Wronskians. In the paper we establish some new results depending on the comparative growth properties of composite entire or meromorphic functions using generalised $L^{*}$-order and generalised $L^{*}$-type and wronskians generated by one of the factors.

\section{Lemmas}

In this section we present some lemmas which will be needed in the sequel.

Lemma 7 (see [7]). If $f$ be meromorphic and $g$ be entire then for all sufficiently large values of $r$,

$$
T(r, f \circ g) \leq\{1+o(1)\} \frac{T(r, g)}{\log M(r, g)} T(M(r, g), f) .
$$

Lemma 8 (see [8]). Let $f$ be meromorphic and $g$ entire and suppose that $0<\mu<\rho_{g} \leq \infty$. Then for a sequence of values of $r$ tending to infinity,

$$
T(r, f \circ g) \geq T\left(\exp \left(r^{\mu}\right), f\right) .
$$

Lemma 9 (see [9]). Let $f$ be a transcendental meromorphic function having the maximum deficiency sum. Then

$$
\lim _{r \rightarrow \infty} \frac{T(r, L(f))}{T(r, f)}=1+k-k \delta(\infty ; f) .
$$

Lemma 10 (see [10]). If $f$ be a transcendental meromorphic function having the maximum deficiency sum. Then the generalised $L^{*}$-order (generalised $L^{*}$-lower order) of $L(f)$ and that of $f$ are the same.

Lemma 11. Let $f$ be a transcendental meromorphic function having the maximum deficiency sum. Then

$$
\begin{gathered}
\sigma_{L(f)}^{[m] L^{*}}=(1+k-k \delta(\infty ; f)) \cdot \sigma_{f}^{[m] L^{*}}, \quad \text { for } m=1, \\
\sigma_{L(f)}^{[m] L^{*}}=\sigma_{f}^{[m] L^{*}}, \quad \text { otherwise. }
\end{gathered}
$$

Proof. By Lemmas 9 and 10 we get that

$$
\begin{aligned}
\sigma_{L(f)}^{L^{*}} & =\limsup _{r \rightarrow \infty} \frac{T(r, L(f))}{\left[r e^{L(r)}\right]^{\rho_{L(f)}^{*}}} \\
& =\lim _{r \rightarrow \infty} \frac{T(r, L(f))}{T(r, f)} \cdot \limsup _{r \rightarrow \infty} \frac{T(r, f)}{\left[r e^{L(r)}\right]^{\rho_{f}^{L^{*}}}} \\
& =(1+k-k \delta(\infty ; f)) \cdot \sigma_{f}^{L^{*}} .
\end{aligned}
$$

Also by Lemma $9, \lim _{r \rightarrow \infty}\left(\log ^{[m-1]} T(r, L(f))\right) /\left(\log ^{[m-1]}\right.$ $T(r, f))$ exists and is equal to 1 for $m>1$.

Therefore

$$
\begin{aligned}
\sigma_{L(f)}^{[m] L^{*}}= & \limsup _{r \rightarrow \infty} \frac{\log ^{[m-1]} T(r, L(f))}{\left[r e^{L(r)}\right]^{\rho_{L(f)}}} \\
= & \lim _{r \rightarrow \infty} \frac{\log ^{[m-1]} T(r, L(f))}{\log ^{[m-1]} T(r, f)} \\
& \cdot \limsup _{r \rightarrow \infty} \frac{\log ^{[m-1]} T(r, f)}{\left[r e^{L(r)}\right]^{\rho_{f}^{L^{*}}}} \\
= & \sigma_{f}^{[m] L^{*}} .
\end{aligned}
$$

Thus the lemma follows. 


\section{Theorems}

In this section we present the main results of the paper.

Theorem 12. Let $f$ be a transcendental meromorphic function having the maximum deficiency sum and $g$ entire such that $0<$ $\lambda_{f}^{[m] L^{*}} \leq \rho_{f}^{[m] L^{*}}<\infty$ where $m$ is any positive integer. Then for any positive real number $A$,

$$
\limsup _{r \rightarrow \infty} \frac{\log ^{[m+1]} T\left(\exp \left(r^{A}\right), f \circ g\right)}{\log ^{[m]} T\left(\exp \left(r^{\mu}\right), L(f)\right)+K(r, g ; L)}=\infty,
$$

where $0<\mu<\rho_{g}$ and

$$
\begin{aligned}
& K(r, g ; L) \\
& \quad= \begin{cases}0 \text { if } r^{\mu}=o\left\{L\left(\exp \left(\exp \left(\mu r^{A}\right)\right)\right)\right\} & \text { as } r \longrightarrow \infty \\
L\left(\exp \left(\exp \left(\mu r^{A}\right)\right)\right) & \text { otherwise. }\end{cases}
\end{aligned}
$$

Proof. Let $0<\mu<\mu^{\prime}<\rho_{g}$. Using the definition of generalised $L^{*}$-lower order we obtain in view of Lemma 8 for a sequence of values of $r$ tending to infinity that

$$
\begin{aligned}
\log ^{[m]} T\left(\exp \left(r^{A}\right), f \circ g\right) \geq \log ^{[m]} T\left(\exp \left(\exp \left(r^{A}\right)\right)^{\mu^{\prime}}, f\right) \\
\text { i.e., } \log { }^{[m]} T\left(\exp \left(r^{A}\right), f \circ g\right) \\
\geq\left(\lambda_{f}^{[m] L^{*}}-\varepsilon\right) \\
\quad \cdot \log \left\{\exp \left(\exp \left(r^{A}\right)\right)^{\mu^{\prime}} \cdot \exp L\left(\exp \left(\exp \left(r^{A}\right)\right)^{\mu^{\prime}}\right)\right\}
\end{aligned}
$$

i.e., $\log ^{[m]} T\left(\exp \left(r^{A}\right), f \circ g\right)$

$$
\geq\left(\lambda_{f}^{[m] L^{*}}-\varepsilon\right) \cdot\left\{\left(\exp \left(r^{A}\right)\right)^{\mu^{\prime}}+L\left(\exp \left(\exp \left(r^{A}\right)\right)^{\mu^{\prime}}\right)\right\}
$$

i.e., $\log ^{[m]} T\left(\exp \left(r^{A}\right), f \circ g\right)$

$$
\geq\left(\lambda_{f}^{[m] L^{*}}-\varepsilon\right)
$$$$
\left\{\left(\exp \left(r^{A}\right)\right)^{\mu^{\prime}}\left(1+\frac{L\left(\exp \left(\exp \left(r^{A}\right)\right)^{\mu^{\prime}}\right)}{\left(\exp \left(r^{A}\right)\right)^{\mu^{\prime}}}\right)\right\}
$$

$$
\begin{gathered}
\text { i.e., } \log ^{[m+1]} T\left(\exp \left(r^{A}\right), f \circ g\right) \\
\geq O(1)+\mu^{\prime} \log \exp \left(r^{A}\right)
\end{gathered}
$$

$$
+\log \left\{1+\frac{L\left(\exp \left(\exp \left(r^{A}\right)\right)^{\mu^{\prime}}\right)}{\left(\exp \left(r^{A}\right)\right)^{\mu^{\prime}}}\right\}
$$

i.e., $\log ^{[m+1]} T\left(\exp \left(r^{A}\right), f \circ g\right)$

$$
\geq O(1)+\mu^{\prime} r^{A}
$$

$$
+\log \left\{1+\frac{L\left(\exp \left(\exp \left(r^{A}\right)\right)^{\mu^{\prime}}\right)}{\left(\exp \left(r^{A}\right)\right)^{\mu^{\prime}}}\right\}
$$

i.e., $\log ^{[m+1]} T\left(\exp \left(r^{A}\right), f \circ g\right)$

$$
\geq O(1)+\mu^{\prime} r^{A}
$$

$$
+\log \left[1+\frac{L\left(\exp \left(\exp \left(\mu^{\prime} r^{A}\right)\right)\right)}{\exp \left(\mu^{\prime} r^{A}\right)}\right]
$$

i.e., $\log ^{[m+1]} T\left(\exp \left(r^{A}\right), f \circ g\right)$

$$
\begin{aligned}
\geq & O(1)+\mu^{\prime} r^{A}+L\left(\exp \left(\exp \left(\mu r^{A}\right)\right)\right) \\
& -\log \left[\exp \left\{L\left(\exp \left(\exp \left(\mu r^{A}\right)\right)\right)\right\}\right] \\
& +\log \left[1+\frac{L\left(\exp \left(\exp \left(\mu^{\prime} r^{A}\right)\right)\right)}{\exp \left(\mu^{\prime} r^{A}\right)}\right]
\end{aligned}
$$

i.e., $\log ^{[m+1]} T\left(\exp \left(r^{A}\right), f \circ g\right)$

$$
\begin{aligned}
& \geq O(1)+\mu^{\prime} r^{A}+L\left(\exp \left(\exp \left(\mu r^{A}\right)\right)\right) \\
& +\log \left[\frac{1}{\exp \left\{L\left(\exp \left(\exp \left(\mu r^{A}\right)\right)\right)\right\}}\right. \\
& \left.\quad+\frac{L\left(\exp \left(\exp \left(\mu^{\prime} r^{A}\right)\right)\right)}{\exp \left\{L\left(\exp \left(\exp \left(\mu r^{A}\right)\right)\right)\right\} \cdot \exp \left(\mu^{\prime} r^{A}\right)}\right]
\end{aligned}
$$

$$
\begin{aligned}
\text { i.e., } \log ^{[m+1]} T\left(\exp \left(r^{A}\right), f \circ g\right) \\
\geq O(1)+\mu^{\prime} r^{(A-\mu)} \cdot r^{\mu} \\
+L\left(\exp \left(\exp \left(\mu r^{A}\right)\right)\right) .
\end{aligned}
$$

Again in view of Lemma 10 we have for all sufficiently large values of $r$ that

$$
\begin{aligned}
\log ^{[m]} & T\left(\exp \left(r^{\mu}\right), L(f)\right) \\
\leq & \left(\rho_{L(f)}^{[m] L^{*}}+\varepsilon\right) \log \left\{\exp \left(r^{\mu}\right) e^{L\left(\exp \left(r^{\mu}\right)\right)}\right\}
\end{aligned}
$$


i.e., $\log ^{[m]} T\left(\exp \left(r^{\mu}\right), L(f)\right)$

$$
\leq\left(\rho_{L(f)}^{[m] L^{*}}+\varepsilon\right)\left\{\log \exp \left(r^{\mu}\right)+L\left(\exp \left(r^{\mu}\right)\right)\right\}
$$

i.e., $\log ^{[m]} T\left(\exp \left(r^{\mu}\right), L(f)\right)$

$$
\begin{gathered}
\leq\left(\rho_{L(f)}^{[m] L^{*}}+\varepsilon\right)\left\{r^{\mu}+L\left(\exp \left(r^{\mu}\right)\right)\right\} \\
\text { i.e., } \frac{\log ^{[m]} T\left(\exp \left(r^{\mu}\right), L(f)\right)-\left(\rho_{L(f)}^{[m] L^{*}}+\varepsilon\right) L\left(\exp \left(r^{\mu}\right)\right)}{\left(\rho_{L(f)}^{[m] L^{*}}+\varepsilon\right)}
\end{gathered}
$$$$
\leq r^{\mu}
$$

Now from (17) and (18) it follows for a sequence of values of $r$ tending to infinity that

$$
\begin{aligned}
\log ^{[m+1]} T\left(\exp \left(r^{A}\right), f \circ g\right) & \\
\geq O(1)+\left(\frac{\mu^{\prime} r^{(A-\mu)}}{\rho_{f}^{[m] L^{*}}+\varepsilon}\right) & {\left[\log ^{[m]} T\left(\exp \left(r^{\mu}\right), L(f)\right)\right.} \\
& \left.-\left(\rho_{f}^{[m] L^{*}}+\varepsilon\right) L\left(\exp \left(r^{\mu}\right)\right)\right] \\
+L\left(\exp \left(\exp \left(\mu r^{A}\right)\right)\right) &
\end{aligned}
$$$$
\text { i.e., } \frac{\log ^{[m+1]} T\left(\exp \left(r^{A}\right), f \circ g\right)}{\log ^{[m]} T\left(\exp \left(r^{\mu}\right), L(f)\right)}
$$$$
\geq \frac{L\left(\exp \left(\exp \left(\mu r^{A}\right)\right)\right)+O(1)}{\log T\left(\exp \left(r^{\mu}\right), L(f)\right)}+\frac{\mu^{\prime} r^{(A-\mu)}}{\rho_{f}^{[m] L^{*}}+\varepsilon}
$$$$
\times\left\{1-\frac{\left(\rho_{f}^{[m] L^{*}}+\varepsilon\right) L\left(\exp \left(r^{\mu}\right)\right)}{\log ^{[m]} T\left(\exp \left(r^{\mu}\right), L(f)\right)}\right\} .
$$

Again from (19) we get for a sequence of values of $r$ tending to infinity that

$$
\begin{aligned}
& \frac{\log ^{[m+1]} T\left(\exp \left(r^{A}\right), f \circ g\right)}{\log ^{[m]} T\left(\exp \left(r^{\mu}\right), L(f)\right)+L\left(\exp \left(\exp \left(\mu r^{A}\right)\right)\right)} \\
& \geq \frac{O(1)-\mu^{\prime} r^{(A-\mu)} L\left(\exp \left(r^{\mu}\right)\right)}{\log ^{[m]} T\left(\exp \left(r^{\mu}\right), L(f)\right)+L\left(\exp \left(\exp \left(\mu r^{A}\right)\right)\right)} \\
& +\frac{\left(\mu^{\prime} r^{(A-\mu)} /\left(\rho_{f}^{[m] L^{*}}+\varepsilon\right)\right) \log ^{[m]} T\left(\exp \left(r^{\mu}\right), L(f)\right)}{\log ^{[m]} T\left(\exp \left(r^{\mu}\right), L(f)\right)+L\left(\exp \left(\exp \left(\mu r^{A}\right)\right)\right)}
\end{aligned}
$$

$$
\begin{aligned}
& +\frac{L\left(\exp \left(\exp \left(\mu r^{A}\right)\right)\right)}{\log ^{[m]} T\left(\exp \left(r^{\mu}\right), L(f)\right)+L\left(\exp \left(\exp \left(\mu r^{A}\right)\right)\right)} \\
& \text { i.e., } \frac{\log ^{[m+1]} T\left(\exp \left(r^{A}\right), f \circ g\right)}{\log ^{[m]} T\left(\exp \left(r^{\mu}\right), L(f)\right)+L\left(\exp \left(\exp \left(\mu r^{A}\right)\right)\right)} \\
& \geq \frac{\left(O(1)-\mu^{\prime} r^{(A-\mu)} L\left(\exp \left(r^{\mu}\right)\right)\right) /\left(L\left(\exp \left(\exp \left(\mu r^{A}\right)\right)\right)\right)}{\left(\log ^{[m]} T\left(\exp \left(r^{\mu}\right), L(f)\right) / L\left(\exp \left(\exp \left(\mu r^{A}\right)\right)\right)\right)+1} \\
& +\left(\left(\left(\frac{\mu^{\prime} r^{(A-\mu)}}{\rho_{f}^{[m] L^{*}}+\varepsilon}\right) \frac{\log ^{[m]} T\left(\exp \left(r^{\mu}\right), L(f)\right)}{L\left(\exp \left(\exp \left(\mu r^{A}\right)\right)\right)}\right)\right. \\
& \left.\times\left(1+\frac{L\left(\exp \left(\exp \left(\mu r^{A}\right)\right)\right)}{\log ^{[m]} T\left(\exp \left(r^{\mu}\right), L(f)\right)}\right)^{-1}\right) \\
& +\frac{1}{1+\log ^{[m]} T\left(\exp \left(r^{\mu}\right), L(f)\right) / L\left(\exp \left(\exp \left(\mu r^{A}\right)\right)\right)} .
\end{aligned}
$$

Case I. If $r^{\mu}=o\left\{L\left(\exp \left(\exp \left(\mu r^{A}\right)\right)\right)\right\}$ then it follows from (20) that

$$
\limsup _{r \rightarrow \infty} \frac{\log ^{[m+1]} T\left(\exp \left(r^{A}\right), f \circ g\right)}{\log ^{[m]} T\left(\exp \left(r^{\mu}\right), L(f)\right)}=\infty .
$$

Case II. $r^{\mu} \neq o\left\{L\left(\exp \left(\exp \left(\mu \mathrm{r}^{A}\right)\right)\right)\right\}$ then the following two subcases may arise.

Subcase (a). If $\left(\exp \left(\exp \left(\mu r^{A}\right)\right)\right)=o\left\{\log ^{[m]} T\left(\exp \left(r^{\mu}\right), L(f)\right)\right\}$, then we get from (21) that

$$
\begin{aligned}
\limsup _{r \rightarrow \infty} & \frac{\log ^{[m+1]} T\left(\exp \left(r^{A}\right), f \circ g\right)}{\log ^{[m]} T\left(\exp \left(r^{\mu}\right), L(f)\right)+L\left(\exp \left(\exp \left(\mu r^{A}\right)\right)\right)} \\
\quad=\infty &
\end{aligned}
$$

Subcase (b). If $L\left(\exp \left(\exp \left(\mu r^{A}\right)\right)\right) \sim \log ^{[m]} T\left(\exp \left(r^{\mu}\right), L(f)\right)$ then

$$
\lim _{r \rightarrow \infty} \frac{L\left\{\exp \left(\exp \left(\mu r^{A}\right)\right)\right\}}{\log ^{[m]} T\left(\exp \left(r^{\mu}\right), L(f)\right)}=1,
$$

and we obtain from (21) that

$$
\begin{aligned}
\limsup _{r \rightarrow \infty} & \frac{\log ^{[m+1]} T\left(\exp \left(r^{A}\right), f \circ g\right)}{\log ^{[m]} T\left(\exp \left(r^{\mu}\right), L(f)\right)+L\left(\exp \left(\exp \left(\mu r^{A}\right)\right)\right)} \\
& =\infty .
\end{aligned}
$$

Combining Cases I and II we obtain that

$$
\limsup _{r \rightarrow \infty} \frac{\log ^{[m+1]} T\left(\exp \left(r^{A}\right), f \circ g\right)}{\log ^{[m]} T\left(\exp \left(r^{\mu}\right), L(f)\right)+K(r, g ; L)}=\infty,
$$


where

$$
\begin{aligned}
K(r, g ; L) & \\
& = \begin{cases}0 \text { if } r^{\mu}=o\{L(\exp (\exp (\mu r A)))\} & \text { as } r \longrightarrow \infty \\
L\left(\exp \left(\exp \left(\mu r^{A}\right)\right)\right) & \text { otherwise. }\end{cases}
\end{aligned}
$$

This proves the theorem.

Theorem 13. Let $f$ be a meromorphic function and $g$ transcendental entire such that $\lambda_{f}^{[m] L^{*}}>0, \rho_{g}^{L^{*}}<\infty$ and $\sum_{a \neq \infty} \delta(a ; g)+\delta(\infty ; g)=2$ where $m \geq 1$. Then for any positive real number $A$,

$$
\limsup _{r \rightarrow \infty} \frac{\log ^{[m+1]} T\left(\exp \left(r^{A}\right), f \circ g\right)}{\log T\left(\exp \left(r^{\mu}\right), L(g)\right)+K(r, f ; L)}=\infty,
$$

where $0<\mu<\rho_{g}$ and

$$
\begin{aligned}
K(r, f ; L) & \\
& = \begin{cases}0 \text { if } r^{\mu}=o\left\{L\left(\exp \left(\exp \left(\mu r^{A}\right)\right)\right)\right\} & \text { as } r \longrightarrow \infty \\
L\left(\exp \left(\exp \left(\mu r^{A}\right)\right)\right) & \text { otherwise. }\end{cases}
\end{aligned}
$$

The proof is omitted because it can be carried out in the line of Theorem 12.

Theorem 14. Let $f$ be a transcendental meromorphicfunction with $\sum_{a \neq \infty} \delta(a ; f)+\delta(\infty ; f)=2$ and $g$ entire such that $\lambda_{f}^{[m] L^{*}}>0,0<\rho_{f}^{[m] L^{*}}<\infty$ and $0<\sigma_{g}^{L^{*}}<\infty$ where $m$ is any positive integer. If $L(M(r, g))=o\left\{r^{\alpha} e^{\alpha L(r)}\right\}$ as $r \rightarrow \infty$ and for some positive $\alpha<\rho_{g}^{L^{*}}$, then

$$
\begin{gathered}
\limsup _{r \rightarrow \infty} \frac{\log ^{[m]} T(r, f \circ g)}{\log ^{[m]} T\left(\exp \left(r e^{L(r)}\right)^{\rho_{g}^{L^{*}}}, L(f)\right)} \\
\leq \frac{\rho_{f}^{[m] L^{*}} \cdot \sigma_{g}^{L^{*}}}{\lambda_{f}^{[m] L^{*}}} .
\end{gathered}
$$

Proof. Since $T(r, g) \leq \log ^{+} M(r, g)$ and by Lemma 7 we get for all sufficiently large values of $r$ that

$$
\begin{aligned}
& \log T(r, f \circ g) \leq \log \{1+o(1)\}+\log T(M(r, g), f) \\
& \text { i.e., } \log ^{[m]} T(r, f \circ g) \leq o(1)+\log ^{[m]} T(M(r, g), f)
\end{aligned}
$$

$$
\text { i.e., } \begin{gathered}
\log ^{[m]} T\left(\exp \left(r e^{L(r)}\right)^{\rho_{g}^{L^{*}}}, L(f)\right) \\
\leq \frac{o(1)+\log ^{[m]} T(M(r, g), f)}{\log ^{[m]} T\left(\exp \left(r e^{L(r)}\right)^{\rho_{g}^{L^{*}}}, L(f)\right)} \\
=\frac{o(1)+\log ^{[m]} T(M(r, g), f)}{\log \left\{M(r, g) e^{L(M(r, g))}\right\}} \\
\cdot \frac{\log M(r, \mathrm{~g})+L(M(r, g))}{\left[r e^{L(r)}\right]^{\rho_{g}^{L^{*}}}} \\
\cdot \frac{\log \left\{\exp \left(r e^{L(r)}\right)^{\rho_{g}^{L^{*}}}\right\}}{\log ^{[m]} T\left(\exp \left(r e^{L(r)}\right)^{\rho_{g}^{L^{*}}}, L(f)\right)}
\end{gathered}
$$

$$
\begin{gathered}
\text { i.e., } \limsup _{r \rightarrow \infty} \frac{\log ^{[m]} T(r, f \circ g)}{\log ^{[m]} T\left(\exp \left(r e^{L(r)}\right)^{\rho_{g}^{L^{*}}}, L(f)\right)} \\
\leq \limsup _{r \rightarrow \infty} \frac{\log ^{[m]} T(M(r, g), f)}{\log \left\{M(r, g) e^{L(M(r, g))}\right\}} \\
\quad \limsup _{r \rightarrow \infty} \frac{\log M(r, g)+L(M(r, g))}{\left[r e^{L(r)}\right]^{\rho_{g}^{L^{*}}}} \\
\cdot \limsup _{r \rightarrow \infty} \frac{\log \left\{\exp \left(r e^{L(r)}\right)^{\rho_{g}^{L^{*}}}\right\}}{\log ^{[m]} T\left(\exp \left(r e^{L(r)}\right)^{\rho_{g}^{L^{*}}}, L(f)\right)} .
\end{gathered}
$$

As $\alpha<\rho_{g}^{L^{*}}$, we can choose $\varepsilon(>0)$ in such a way that $\alpha<$ $\rho_{g}^{L^{*}}-\varepsilon$ and since $L(M(r, g))=o\left(r^{\alpha} e^{\alpha L(r)}\right)$ as $r \rightarrow \infty$, we obtain that

$$
\lim _{r \rightarrow \infty} \frac{L(M(r, g))}{\left[r e^{L(r)}\right]^{\rho_{g}^{*}-\varepsilon}}=0 .
$$

Now from (32) and (33) and in view of Lemma 10 it follows that

$$
\begin{gathered}
\limsup _{r \rightarrow \infty} \frac{\log ^{[m]} T(r, f \circ g)}{\log ^{[m]} T\left(\exp \left(r e^{L(r)}\right)^{\rho_{g}^{L^{*}}}, L(f)\right)} \\
\leq \rho_{f}^{[m] L^{*}} \cdot \sigma_{g}^{L^{*}} \cdot \frac{1}{\lambda_{L(f)}^{[m] L^{*}}}
\end{gathered}
$$

$$
\begin{aligned}
& \text { i.e., } \limsup _{r \rightarrow \infty} \frac{\log ^{[m]} T(r, f \circ g)}{\log ^{[m]} T\left(\exp \left(r e^{L(r)}\right)^{\rho_{g}^{L^{*}}}, L(f)\right)} \\
& \leq \rho_{f}^{[m] L^{*}} \cdot \sigma_{g}^{L^{*}} \cdot \frac{1}{\lambda_{f}^{[m] L^{*}}} .
\end{aligned}
$$

Thus the theorem is established. 
In the line of Theorem 14 the following theorem can be proved.

Theorem 15. Let $f$ be meromorphic and $g$ transcendental entire having the maximum deficiency sum such that $\lambda_{g}^{L^{*}}>0$, $0<\rho_{f}^{[m] L^{*}}<\infty$ and $0<\sigma_{g}^{L^{*}}<\infty$ where $m \geq 1$. If $L(M(r, g))=o\left\{r^{\alpha} e^{\alpha L(r)}\right\}$ as $r \rightarrow \infty$ and for some positive $\alpha<\rho_{g}^{L^{*}}$, then

$$
\begin{gathered}
\limsup _{r \rightarrow \infty} \frac{\log ^{[m]} T(r, f \circ g)}{\log T\left(\exp \left(r e^{L(r)}\right)^{\rho_{g}^{L^{*}}}, L(g)\right)} \\
\leq \frac{\rho_{f}^{[m] L^{*}} \cdot \sigma_{g}^{L^{*}}}{\lambda_{g}^{L^{*}}} .
\end{gathered}
$$

The proof is omitted.

Theorem 16. Let $f$ be a transcendental meromorphic function such that $\rho_{f}^{[n] L^{*}}<\infty$ with $n \geq 1$ and $\sum_{a \neq \infty} \delta(a ; f)+$ $\delta(\infty ; f)=2$. Also let $g$ be entire. If $\lambda_{f \circ g}^{[m] L^{*}}=\infty$ for $m \geq 1$ then

$$
\lim _{r \rightarrow \infty} \frac{\log ^{[m]} T(r, f \circ g)}{\log ^{[n]} T(r, L(f))}=\infty
$$

Proof. Let us suppose that the conclusion of the theorem does not hold.Then we can find a constant $\beta>0$ such that for a sequence of values of $r$ tending to infinity

$$
\log ^{[m]} T(r, f \circ g) \leq \beta \log ^{[n]} T(r, L(f)) .
$$

Again from the definition of $\rho_{L(f)}^{L^{*}}$ it follows that for all sufficiently large values of $r$ and in view of Lemma 10,

$$
\begin{aligned}
& \log ^{[n]} T(r, L(f)) \leq\left(\rho_{L(f)}^{[n] L^{*}}+\varepsilon\right) \log \left(r e^{L(r)}\right) \\
& \text { i.e., } \log ^{[n]} T(r, L(f)) \leq\left(\rho_{f}^{[n] L^{*}}+\varepsilon\right) \log \left(r e^{L(r)}\right) .
\end{aligned}
$$

Thus from (37) and (38) we have for a sequence of values of $r$ tending to infinity that

$$
\begin{aligned}
& \log ^{[m]} T(r, f \circ g) \leq \beta\left(\rho_{f}^{[n] L^{*}}+\varepsilon\right) \log \left(r e^{L(r)}\right) \\
& \text { i.e., } \frac{\log ^{[m]} T(r, f \circ g)}{\log \left(r e^{L(r)}\right)} \leq \frac{\beta\left(\rho_{f}^{[n] L^{*}}+\varepsilon\right) \log \left(r e^{L(r)}\right)}{\log \left(r e^{L(r)}\right)} \\
& \text { i.e., } \liminf _{r \rightarrow \infty} \frac{\log ^{[m]} T(r, f \circ g)}{\log r}=\lambda_{f \circ g}^{[m] L^{*}}<\infty .
\end{aligned}
$$

This is a contradiction. This proves the theorem.

Remark 17. Theorem 16 is also valid with "limit superior" instead of "limit" if $\lambda_{f \circ g}^{[m] L^{*}}=\infty$ is replaced by $\rho_{f \circ g}^{[m] L^{*}}=\infty$ and the other conditions remain the same.
Corollary 18. Under the assumptions of Theorem 16 or Remark 17,

$$
\limsup _{r \rightarrow \infty} \frac{\log ^{[m-1]} T(r, f \circ g)}{\log ^{[n-1]} T(r, L(f))}=\infty .
$$

Proof. From Theorem 16 or Remark 17 we obtain for all sufficiently large values of $r$ and for $K>1$,

$$
\begin{gathered}
\log ^{[m]} T(r, f \circ g)>K \log ^{[n]} T(r, L(f)), \\
\text { i.e., } \log ^{[m-1]} T(r, f \circ g)>\left\{\log ^{[n-1]} T(r, L(f))\right\}^{K},
\end{gathered}
$$

from which the corollary follows.

Remark 19. The condition $\lambda_{f \circ g}^{[m] L^{*}}=\infty$ in Theorem 16 and Corollary 18 is necessary which is evident from the following example.

Example 20. Let $f=\exp z, g=z, m=n=1$, and $L(r)=$ $(1 / p) \exp (1 / r)$ where $p$ is any positive real number.

Then

$$
\begin{aligned}
& \rho_{f}^{L^{*}}=1<\infty, \quad \lambda_{f \circ g}^{L^{*}}=1<\infty, \\
& \sum_{a \neq \infty} \delta(a ; f)+\delta(\infty ; f)=2 .
\end{aligned}
$$

Now taking $a_{1}=1$ and $a_{2}=\cdots=a_{k}=0$ we get from Definition 2 that

$$
L(f)=\left|\begin{array}{ll}
a_{1} & f \\
a_{1}^{\prime} & f^{\prime}
\end{array}\right|=\left|\begin{array}{ll}
1 & \exp z \\
0 & \exp z
\end{array}\right|=\exp z
$$

Now

$$
\begin{aligned}
& T(r, f \circ g)=T(r, \exp z)=\frac{r}{\pi} \\
& T(r, L(f))=T(r, \exp z)=\frac{r}{\pi}
\end{aligned}
$$

Therefore

$$
\begin{gathered}
\lim _{r \rightarrow \infty} \frac{\log T(r, f \circ g)}{\log T(r, L(f))}=\lim _{r \rightarrow \infty} \frac{\log r+O(1)}{\log r+O(1)}=1, \\
\lim _{r \rightarrow \infty} \frac{T(r, f \circ g)}{T(r, L(f))}=\lim _{r \rightarrow \infty} \frac{(r / \pi)}{(r / \pi)}=1,
\end{gathered}
$$

which is a contradiction.

Remark 21. Considering $f=\exp z, g=z, m=n=1, A=$ $1, L(r)=(1 / p) \exp (1 / r)$ for any positive real number $p$ and taking $a_{1}=1, a_{2}=\cdots=a_{k}=0$ in Definition 2, one can easily verify that the condition $\rho_{f \circ g}^{[m] L^{*}}=\infty$ in Remark 17 and Corollary 18 is essential.

Theorem 22. Let $f$ be meromorphic and $g$ transcendental entire such that $\rho_{g}^{[n] L^{*}}<\infty$ with $n \geq 1$ and $\lambda_{f \circ g}^{[m] L^{*}}=\infty$ where 
$m$ is any positive integer. Also let $\sum_{a \neq \infty} \delta(a ; g)+\delta(\infty ; g)=2$. Then

$$
\lim _{r \rightarrow \infty} \frac{\log ^{[m]} T(r, f \circ g)}{\log ^{[n]} T(r, L(g))}=\infty
$$

We omit the proof of Theorem 22 because it can be carried out in the line of Theorem 16.

Remark 23. Theorem 22 is also valid with "limit superior" instead of "limit" if $\lambda_{f \circ g}^{[m] L^{*}}=\infty$ is replaced by $\rho_{f \circ g}^{[m] L^{*}}=\infty$ and the other conditions remain the same.

In the line of Corollary 18 we may easily verify the following.

Corollary 24. Under the assumptions of Theorem 22 or Remark 19,

$$
\limsup _{r \rightarrow \infty} \frac{\log ^{[m-1]} T(r, f \circ g)}{\log ^{[n-1]} T(r, L(g))}=\infty
$$

Remark 25. Taking $f=z, g=\exp z, m=n=1, A=1$, $L(r)=(1 / p) \exp (1 / r)$ for any positive real number $p$ and choosing $a_{1}=1, a_{2}=\cdots=a_{k}=0$ in Definition 2 we may establish the necessity of the conditions $\lambda_{f \circ g}^{[m] L^{*}}=\infty$ and $\rho_{f \circ g}^{[m] L^{*}}=\infty$, respectively, in Theorem 22, Remark 23, and Corollary 24.

Theorem 26. Let $f$ be meromorphic function and $g$ entire function having the maximum deficiency sum such that $(i) 0<$ $\rho_{f}^{[m] L^{*}}<\infty$ and $(i i) \sigma_{g}^{L^{*}}<\infty$ where $m$ is any positive integer. Then

(a) if $L(M(r, g))=o\{T(r, L(g))\}$ then

$$
\liminf _{r \rightarrow \infty} \frac{\log ^{[m]} T(r, f \circ g)}{T(r, L(g))+L(M(r, g))} \leq \frac{\rho_{f}^{[m] L^{*}}}{(1+k-k \delta(\infty ; g))}
$$

and $(b)$ if $T(r, L(g))=o\{L(M(r, g))\}$ then

$$
\liminf _{r \rightarrow \infty} \frac{\log ^{[m]} T(r, f \circ g)}{T(r, L(g))+L(M(r, g))} \leq \rho_{f}^{[m] L^{*}}
$$

Proof. Since $T(r, g) \leq \log ^{+} M(r, g)$ in view of Lemma 7 we obtain for all sufficiently large values of $r$ that

$$
\begin{aligned}
\log T & (r, f \circ g) \\
& \leq \log \{1+o(1)\}+\log T(M(r, g), f)
\end{aligned}
$$

$$
\begin{aligned}
& \text { i.e., } \log ^{[m]} T(r, f \circ g) \\
& \leq o(1)+\log ^{[m]} T(M(r, g), f) \\
& \text { i.e., } \log ^{[m]} T(r, f \circ g) \\
& \leq o(1)+\left(\rho_{f}^{[m] L^{*}}+\varepsilon\right) \\
& \quad \times\{\log M(r, g)+L(M(r, g))\} .
\end{aligned}
$$

Using the definition of $L^{*}$-type we obtain from (50) for all sufficiently large values of $r$ that

$$
\begin{aligned}
\log ^{[m]} T(r, f \circ g) \leq & o(1) \\
& +\left(\rho_{f}^{[m] L^{*}}+\varepsilon\right)\left(\sigma_{g}^{L^{*}}+\varepsilon\right)\left\{r e^{L(r)}\right\}^{\rho_{g}^{L^{*}}} \\
& +\left(\rho_{f}^{[m] L^{*}}+\varepsilon\right) L(M(r, g)) .
\end{aligned}
$$

Again from the definition of $L^{*}$-type and in view of Lemmas 10 and 11 we get for a sequence of values of $r$ tending to infinity that

$$
\begin{aligned}
& T(r, L(g)) \geq\left(\sigma_{L(g)}^{L^{*}}-\varepsilon\right)\left\{r e^{L(r)}\right\}^{\rho_{L(g)}^{L^{*}}} \\
& \text { i.e., } T(r, L(g)) \\
& \qquad \geq\left\{(1+k-k \delta(\infty ; g)) \sigma_{g}^{L^{*}}-\varepsilon\right\}\left\{r e^{L(r)}\right\}^{\rho_{g}^{L^{*}}} \\
& \text { i.e., }\left\{r e^{L(r)}\right\}^{\rho_{g}^{L^{*}}} \leq \frac{T(r, L(g))}{\left((1+k-k \delta(\infty ; g)) \sigma_{g}^{L^{*}}-\varepsilon\right)} .
\end{aligned}
$$

Now from (51) and (52) it follows for a sequence of values of $r$ tending to infinity that

$$
\begin{aligned}
\log ^{[m]} T(r, f \circ g) \\
\leq o(1)+\left(\rho_{f}^{[m] L^{*}}+\varepsilon\right)\left(\sigma_{g}^{L^{*}}+\varepsilon\right) \\
\quad \times \frac{T(r, L(g))}{\left((1+k-k \delta(\infty ; g)) \sigma_{g}^{L^{*}}-\varepsilon\right)} \\
\quad+\left(\rho_{f}^{[m] L^{*}}+\varepsilon\right) L(M(r, g)) \\
\text { i.e., } \frac{\log ^{[m]} T(r, f \circ g)}{T(r, L(g))+L(M(r, g))}
\end{aligned}
$$




$$
\begin{aligned}
& \leq \frac{o(1)}{T(r, L(g))+L(M(r, g))} \\
& +\left(\left(\frac{\left(\rho_{f}^{[m] L^{*}}+\varepsilon\right)\left(\sigma_{g}^{L^{*}}+\varepsilon\right)}{\left((1+k-k \delta(\infty ; g)) \sigma_{g}^{L^{*}}-\varepsilon\right)}\right)\right. \\
& \left.\quad \times\left(1+\frac{L(M(r, g))}{T(r, L(g))}\right)^{-1}\right) \\
& +\frac{\left(\rho_{f}^{[m] L^{*}}+\varepsilon\right)}{1+(T(r, L(g)) / L(M(r, g)))} .
\end{aligned}
$$

If $L(M(r, g))=o\{T(r, L(g))\}$ then from (53) we get that

$$
\begin{aligned}
\liminf _{r \rightarrow \infty} \frac{\log ^{[m]} T(r, f \circ g)}{T(r, L(g))+L(M(r, g))} \\
\quad \leq \frac{\left(\rho_{f}^{[m] L^{*}}+\varepsilon\right)\left(\sigma_{g}^{L^{*}}+\varepsilon\right)}{(1+k-k \delta(\infty ; g))\left(\sigma_{g}^{L^{*}}-\varepsilon\right)} .
\end{aligned}
$$

Since $\varepsilon(>0)$ is arbitrary it follows from above that

$$
\begin{gathered}
\liminf _{r \rightarrow \infty} \frac{\log ^{[m]} T(r, f \circ g)}{T(r, L(g))+L(M(r, g))} \\
\leq \frac{\rho_{f}^{[m] L^{*}}}{(1+k-k \delta(\infty ; g))} .
\end{gathered}
$$

Thus the first part of Theorem 26 follows.

Again if $T(r, L(g))=o\{L(M(r, g))\}$ then from (53) it follows that

$$
\liminf _{r \rightarrow \infty} \frac{\log ^{[m]} T(r, f \circ g)}{T(r, L(g))+L(M(r, g))} \leq\left(\rho_{f}^{[m] L^{*}}+\varepsilon\right) .
$$

As $\varepsilon(>0)$ is arbitrary we obtain from the above that

$$
\liminf _{r \rightarrow \infty} \frac{\log ^{[m]} T(r, f \circ g)}{T(r, L(g))+L(M(r, g))} \leq \rho_{f}^{[m] L^{*}} .
$$

Thus the second part of Theorem 26 follows.

Theorem 27. Let $f$ be a transcendental meromorphic function having the maximum deficiency sum and $g$ entire such that (i) $0<\rho_{f}^{[m] L^{*}}<\infty$, (ii) $\rho_{f}^{[m] L^{*}}=\rho_{g}^{L^{*}}$, (iii) $\sigma_{g}^{L^{*}}<\infty$ and (iv) $0<\sigma_{f}^{[m] L^{*}}<\infty$ where $m \geq 1$. Then (a) if $L(M(r, g))=o\left\{r^{\alpha} e^{\alpha L(r)}\right\}$ as $r \rightarrow \infty$ and for some positive $\alpha<\rho_{f}^{[m] L^{*}}$ then

$$
\begin{aligned}
& \liminf _{r \rightarrow \infty} \frac{\log T(r, f \circ g)}{T(r, L(f))+L(M(r, g))} \\
& \quad \leq \frac{\rho_{f}^{L^{*}} \cdot \sigma_{g}^{L^{*}}}{(1+k-k \delta(\infty ; f)) \cdot \sigma_{f}^{L^{*}}}, \\
& \liminf _{r \rightarrow \infty} \frac{\log ^{[m]} T(r, f \circ g)}{\log ^{[m-1]} T(r, L(f))+L(M(r, g))} \\
& \leq \frac{\rho_{f}^{[m] L^{*}} \cdot \sigma_{g}^{L^{*}}}{\sigma_{f}^{[m] L^{*}}}, \quad \text { for } m>1,
\end{aligned}
$$

and $(b)$ if $\log ^{[m-1]} T(r, L(f))=o\{L(M(r, g))\}$ then

$$
\liminf _{r \rightarrow \infty} \frac{\log ^{[m]} T(r, f \circ g)}{\log ^{[m-1]} T(r, L(f))+L(M(r, g))} \leq \rho_{f}^{[m] L^{*}} .
$$

Proof. In view of condition (ii) we obtain from (51) for all sufficiently large values of $r$ that

$$
\begin{aligned}
\log ^{[m]} T(r, f \circ g) \leq & o(1)+\left(\rho_{f}^{[m] L^{*}}+\varepsilon\right) \\
& \times\left(\sigma_{g}^{L^{*}}+\varepsilon\right)\left\{r e^{L(r)}\right\}^{\rho_{f}^{[m] L^{*}}} \\
& +\left(\rho_{f}^{[m] L^{*}}+\varepsilon\right) L(M(r, g)) .
\end{aligned}
$$

Again from the definition of $L^{*}$-type and in view of Lemma 10 we get for a sequence of values of $r$ tending to infinity that

$$
\begin{aligned}
& \log ^{[m-1]} T(r, L(f)) \\
& \qquad\left(\sigma_{L(f)}^{[m] L^{*}}-\varepsilon\right)\left\{r e^{L(r)}\right\}^{\rho_{L(f)}^{[m] L^{*}}} \\
& \text { i.e., } \log ^{[m-1]} T(r, L(f)) \\
& \quad \geq\left(\sigma_{L(f)}^{[m] L^{*}}-\varepsilon\right)\left\{r e^{L(r)}\right\}_{f}^{\rho_{f}^{[m] L^{*}}} \\
& \text { i.e., }\left\{r e^{L(r)}\right\}_{f}^{\rho_{f}^{[m] L^{*}}} \leq \frac{\log ^{[m-1]} T(r, L(f))}{\left(\sigma_{L(f)}^{[m] L^{*}}-\varepsilon\right)} .
\end{aligned}
$$


Now from (60) and (61) it follows for a sequence of values of $r$ tending to infinity that

$$
\begin{aligned}
& \log ^{[m]} T(r, f \circ g) \\
& \leq o(1)+\left(\rho_{f}^{[m] L^{*}}+\varepsilon\right)\left(\sigma_{g}^{L^{*}}+\varepsilon\right) \\
& \times \frac{\log ^{[m-1]} T(r, L(f))}{\left(\sigma_{L(f)}^{[m] L^{*}}-\varepsilon\right)} \\
& +\left(\rho_{f}^{[m] L^{*}}+\varepsilon\right) L(M(r, g)) \\
& \text { i.e., } \frac{\log ^{[m]} T(r, f \circ g)}{\log ^{[m-1]} T(r, L(f))+L(M(r, g))} \\
& \leq \frac{o(1)}{\log ^{[m-1]} T(r, L(f))+L(M(r, g))} \\
& +\left(\left(\frac{\left(\rho_{f}^{[m] L^{*}}+\varepsilon\right)\left(\sigma_{g}^{L^{*}}+\varepsilon\right)}{\left(\sigma_{L(f)}^{[m] L^{*}}-\varepsilon\right)}\right)\right. \\
& \left.\times\left(1+\frac{L(M(r, g))}{\log ^{[m-1]} T(r, L(f))}\right)^{-1}\right) \\
& +\frac{\left(\rho_{f}^{[m] L^{*}}+\varepsilon\right)}{1+\left(\log ^{[m-1]} T(r, L(f)) / L(M(r, g))\right)} .
\end{aligned}
$$

If $L(M(r, g))=o\left\{\log ^{[m-1]} T(r, L(f))\right\}$ then from (62) we get that

$$
\begin{aligned}
\liminf _{r \rightarrow \infty} & \frac{\log ^{[m]} T(r, f \circ g)}{\log ^{[m-1]} T(r, L(f))+L(M(r, g))} \\
\leq & \frac{\left(\rho_{f}^{[m] L^{*}}+\varepsilon\right)\left(\sigma_{g}^{L^{*}}+\varepsilon\right)}{\left(\sigma_{L(f)}^{[m] L^{*}}-\varepsilon\right)} .
\end{aligned}
$$

Since $\varepsilon(>0)$ is arbitrary, it follows from the above that

$$
\begin{aligned}
& \liminf _{r \rightarrow \infty} \frac{\log ^{[m]} T(r, f \circ g)}{\log ^{[m-1]} T(r, L(f))+L(M(r, g))} \\
& \quad \leq \frac{\rho_{f}^{[m] L^{*}} \cdot \sigma_{g}^{L^{*}}}{\sigma_{L(f)}^{[m] L^{*}}} .
\end{aligned}
$$

Now in view of Lemma 11 we get from the above that

$$
\begin{aligned}
& \liminf _{r \rightarrow \infty} \frac{\log T(r, f \circ g)}{T(r, L(f))+L(M(r, g))} \\
& \quad \leq \frac{\rho_{f}^{L^{*}} \cdot \sigma_{g}^{L^{*}}}{(1+k-k \delta(\infty ; f)) \cdot \sigma_{f}^{L^{*}}}, \quad \text { for } m=1, \\
& \liminf _{r \rightarrow \infty} \frac{\log ^{[m]} T(r, f \circ g)}{\log ^{[m-1]} T(r, L(f))+L(M(r, g))} \\
& \quad \leq \frac{\rho_{f}^{[m] L^{*}} \cdot \sigma_{g}^{L^{*}}}{\sigma_{f}^{[m] L^{*}}}, \quad \text { for } m>1 .
\end{aligned}
$$

Thus the first part of Theorem 27 follows.

Again if $\log ^{[m-1]} T(r, L(f))=o\{L(M(r, g))\}$ then from (62) it follows that

$$
\liminf _{r \rightarrow \infty} \frac{\log ^{[m]} T(r, f \circ g)}{\log ^{[m-1]} T(r, L(f))+L(M(r, g))} \leq\left(\rho_{f}^{[m] L^{*}}+\varepsilon\right) .
$$

As $\varepsilon(>0)$ is arbitrary, we obtain from the above that

$$
\liminf _{r \rightarrow \infty} \frac{\log ^{[m]} T(r, f \circ g)}{\log ^{[m-1]} T(r, L(f))+L(M(r, g))} \leq \rho_{f}^{[m] L^{*}} .
$$

Thus the second part of Theorem 27 follows.

\section{References}

[1] W. K. Hayman, Meromorphic Functions, The Clarendon Press, Oxford, UK, 1964.

[2] G. Valiron, Lectures on the General Theory of Integral Functions, Chelsea, 1949.

[3] S. K. Singh and G. P. Barker, "Slowly changing functions and their applications," Indian Journal of Mathematics, vol. 19, no. 1, pp. 1-6, 1977.

[4] D. Somasundaram and R. Thamizharasi "note on the entire functions of L-bounded index and L-type," Indian Journal of Pure and Applied Mathematics, vol. 19, no. 3, pp. 284-293, 1988.

[5] T. V. Lakshminarasimhan, "A note on entire functions of bounded index," Journal of the Indian Mathematical Society, vol. 38, pp. 43-49, 1974.

[6] I. Lahiri and N. R. Bhattacharjee, "Functions of L-bounded index and of non-uniform L-bounded index," Indian Journal of Mathematics, vol. 40, pp. 43-57, 1998.

[7] W. Bergweiler, "On the Nevanlinna Characteristic of a composite function," Complex Variables, vol. 10, pp. 225-236, 1988.

[8] W. Bergweiler, "On the growth rate of composite meromorphic functions," Complex Variables, vol. 14, pp. 187-196, 1990.

[9] I. Lahiri and A. Banerjee Value Distribution of a Wronskian, Portugaliae Mathematica, vol. 61, Nova Série, 2004.

[10] S. K. Datta and T. Biswas, "On the L-(p, q)-th order of wronskians," International Journal of Pure and Applied Mathematics, vol. 50, no. 3, pp. 373-378, 2009. 


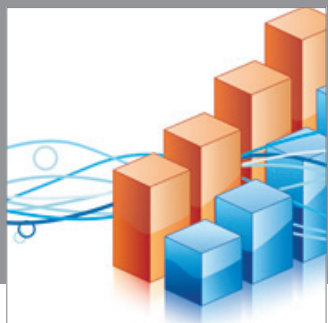

Advances in

Operations Research

mansans

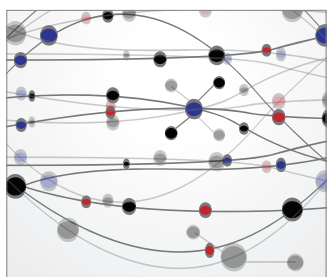

The Scientific World Journal
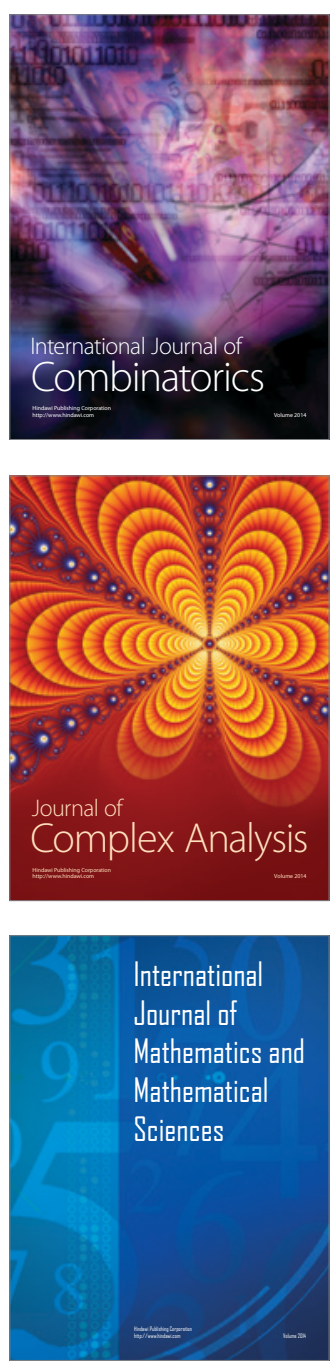
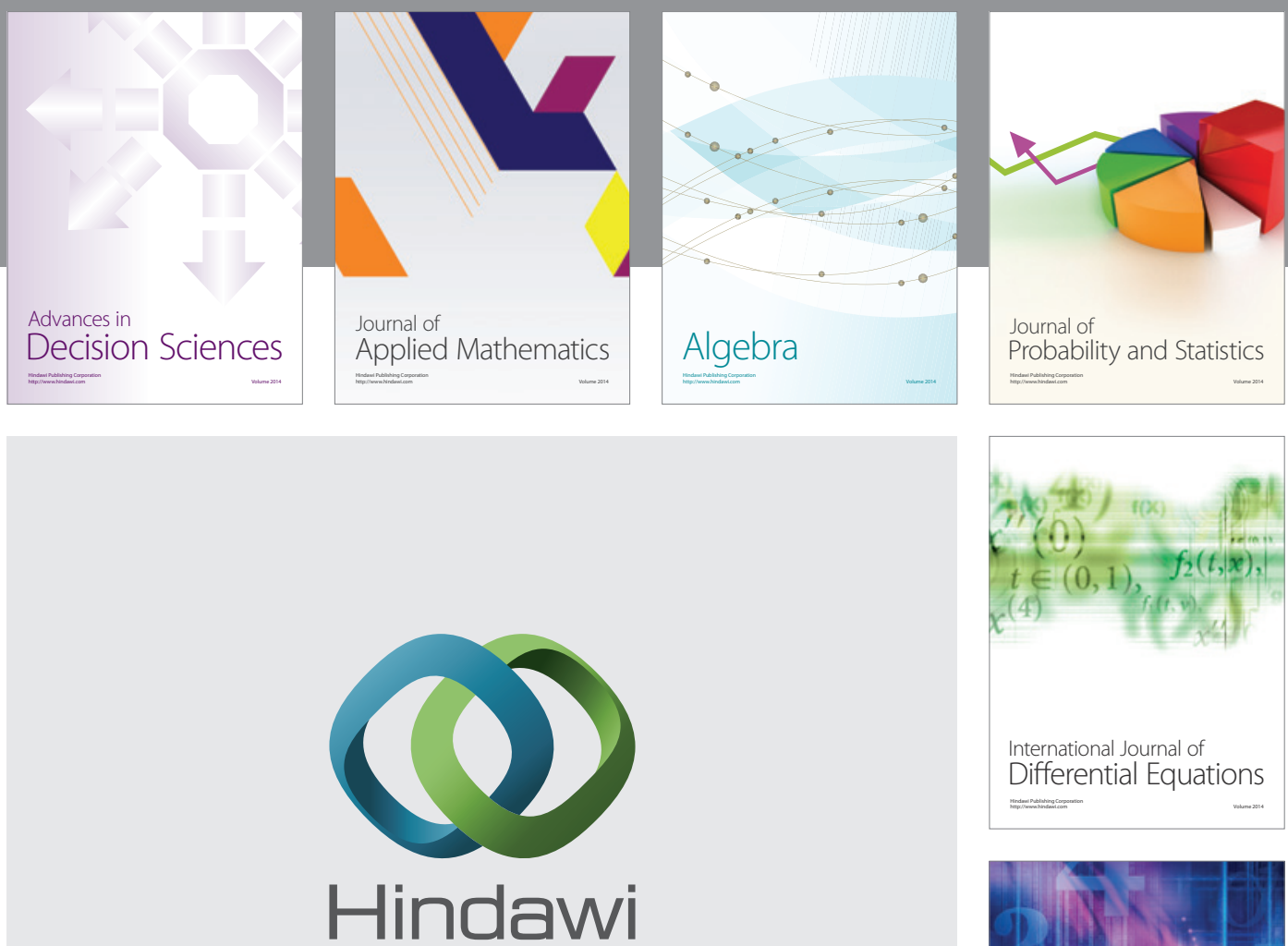

Submit your manuscripts at http://www.hindawi.com
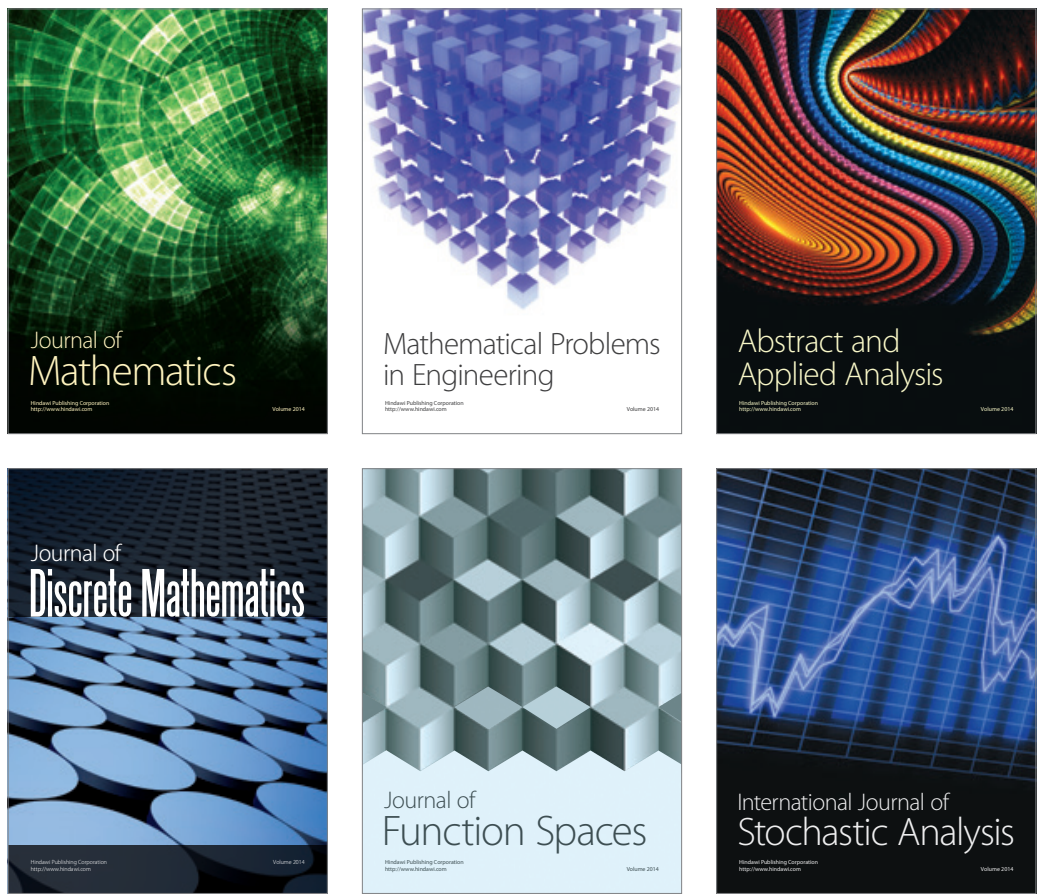

Journal of

Function Spaces

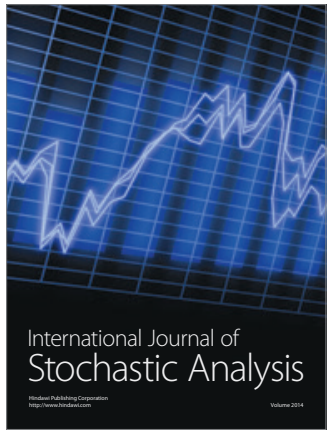

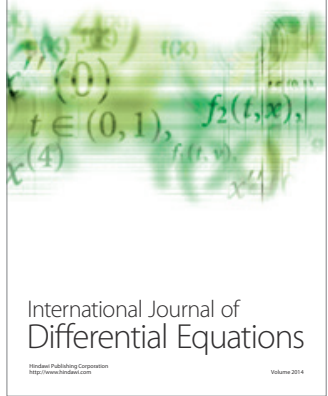
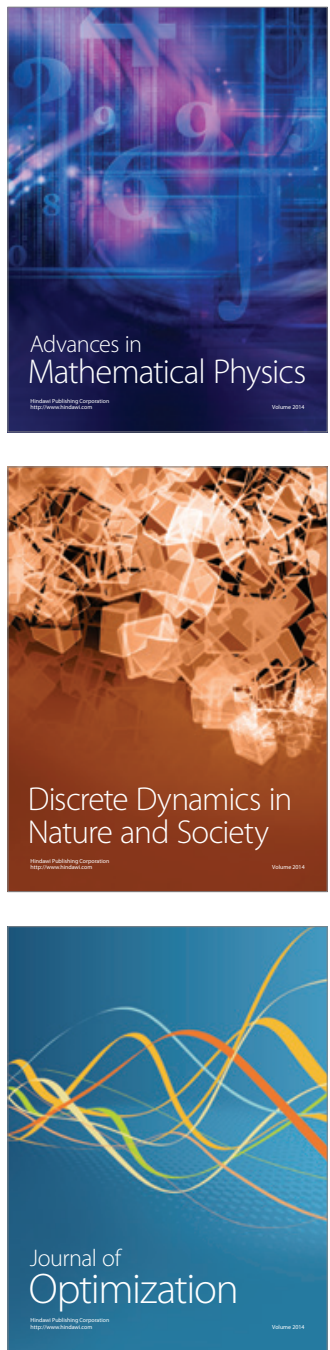